Technology and Innovation

Nancy Esperanza Olarte López Universidad Militar

Nueva Granada

Carlos Antonio Orrego Muñoz Universidad Militar Nueva Granada

\section{Spectra Analysis}

\section{of an Amateur}

\section{Radio Antenna Design *}

\section{y)}

Gopen access CIEnCIA y poder AÉreo

ISSN 1909-7050/ E-ISSN 2389-240

200-215

Citación: Olarte, N., Orrego, C.. y Echeverry, G. (2019). Análisis espectral del

Doi: https://doi.org/10.18667/cienciaypoderaereo.641

Nancy Esperanza olarte López

Ingeniera en Telecomunicaciones, Especialista

a la Educacaión, Magister en Tecnologias de

Informacin AplicadralaEducación.

nancy.olarte@unimilitar.edu.co

do?cod_rh $=0000812781$

ORCID: http://orcid.

Îndice $\mathrm{H}: 8$

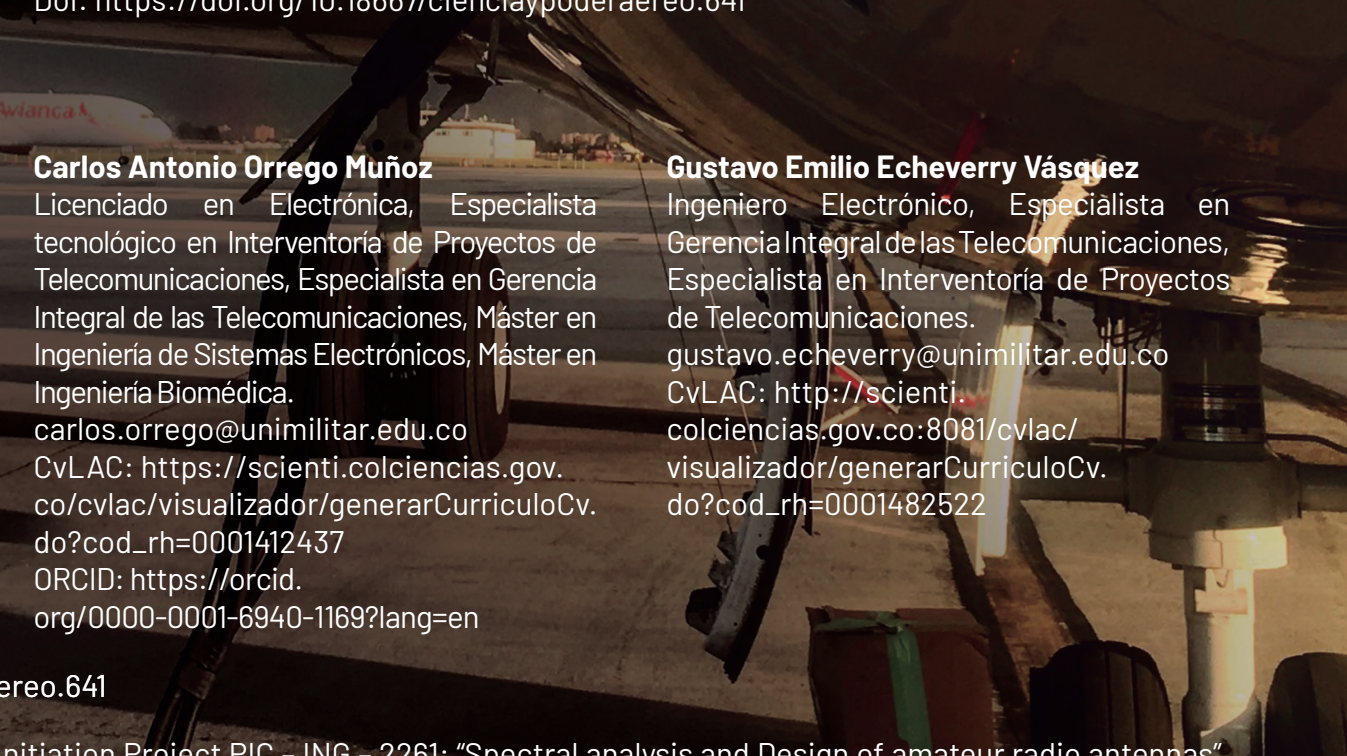

Doi:https://doi.org/10.18667/cienciaypoderaereo.64

1 * Research article based on the Scientific Initiation Project PIC - ING - 2261: "Spectral analysis and Design of amateur radio antennas". eva Granada.

\section{Abstract}

Nowadays, amateur radio can be, in extreme cases, one of the last remaining communication resources. Its worldwide relevance and number of fosources l lsworld re of local amateur radio organizations.

On the other hand, knowing how to design different kinds of amateur radio antennas is of great interest not only to the communities that make up such organizations but also to those in the academic field particuly as it concerns practical experienfield, particularly as it concerns practical experience with communications systems, and in regard to
emergency or disaster situations.

This research shows the process to design an antenna prototype that can be made using easy-tofind elements. Furthermore, it shows the transmission and reception tests carried out after the construction of the prototype, with the aim of con- ducting a spectrum analysis within a local setting. First, the text offers a brief explanation of the importance of amateur radio in communication; next, there is a description of the antenna type chosen and the methods applied to its construction; then, there is a comparative analysis of the different tests carried out at various transmission powers and at different distances in a local scenario.

\section{Key Words:}

Antenna, Communication, Power, Radio Amateur, Reception, Transmission, Yagi-Uda 
Nancy Esperanza Olarte López Universidad Militar Nueva Granada Carlos Antonio Orrego Muñoz
Universidad Militar Nueva Granada

\section{Gustavo Emilio Echeverry Vásquez}

Anális espectial

\section{do desenho}

\section{de uma antena de radioamador *}

\section{Gopen access CIENCIA y PODER AÉREO}

Julio-Diciemb

Citación: Olarte, N., Orrego, C. y Echeverry, G. (2019). Análisis espectral del

Deino de

Doi: https://doi.org/10.18667/cienciaypoderaereo.641

\section{Nancy Esperanza Olarte López}

Ingeniera en Telecomunicaciones, Especialista

a lecolucación. Magister en Tecnologias de

Información Aplicadas a la Educación.

\section{nancy.olarte@unimilitar.edu.co}

\section{co/cvlac/visualizador/generarCurriculocv.}

(10000-0001-7029-4006?lang=es

Índice H: 8

Doi:https://doi.org/10.18667/cienciaypoderaereo.641

1 *Artigo de pesquisa, derivado do Projeto de Iniciaçâao Cientifica PIC - ING - 2261: "Desenho e análise espectral de antenas de radioamat (Grupo de Pesquisa e inovacão Tecnológica em Eletrônica e Comunicaçōes). Financiado pela Universidade Militar Nueva Granada.

\section{Resumo}

0 radioamador hoje em dia é um dos últimos recursos de comunicação em casos extremos. A sua importância no âmbito mundial e número de seguidores dia a dia aumenta, contribuindo à formação de ligas de radioamadores locais.

Por outro lado, o conhecer como desenhar tipos de antenas de radioamador também é de grande interesse para não só as comunidades que conformam as ligas, como também se incrementa no âmbito acadêmico, particularmente na prática em um sistema de comunicação e sua consideração em entornos de emergencias e desastres.

Esta pesquisa evidencia como se propôs o desenho de um protótipo de antena a partir de elementos de fácil acesso e posterior a sua construção, as provas de transmiscão e recepcão para sua análise espectral em um ambiente local.

Em primeiro lugar, brevemente se explica a importância do radioamador nas comunicações, logo se realiza uma descricão sobre o tipo de antena escoliza una decto sobre a tipo de antena escoliza e os métodos empregados para a sua compaço, posteriormente se realiza uma análise cor diferentivas diferentes provas realizadas sob

Palavras-chave:

antena, comunicação, potência, radioamador, recepção, transmissão, Yagi-Uda. 
Carlos Antonio Orrego Muñoz Carlos Antonio Orrec
Universidad Milita Nueva Granada Nueva Granada

Anólis. Análisi espectral

\section{del diseño}

\section{de una antena de} radio afición *

Gopen access ciencia y poder AÉReo

ISSN 1909-7050 / E- ISSN 2389-2468 / Volumen 14 (2)
Julio-Diciembre de 2019/ Colombia/ Pp. 200-215

Citación: Olarte, N., Orrego, C. y Echeverry, G. (2019). Análisis espectral del

(200-215

Doi: https://doi.org/10.18667/cienciaypoderaereo.641

Nancy Esperanza olarte López

Ingeniera en Telecomunicaciones, Especialista
en Tecnologias de la Información Aplicadas

en ecucación Magister en Tecnologias de

Información Aplicadas a la Educación.

\section{nancy.olarte@unimilitar.edu.co}

\section{co/cylac/visualizador/generarCurriculocv.}

Doi:https://doi.org/10.18667/cienciaypoderaere0.641

1 *Articulo de investigación, derivado del Proyecto de Iniciación Cientifica PIC - ING - 2261: "Diseño y análisis espectral de antenas de

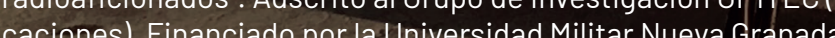

\section{Resumen}

La radio afición hoy en día es uno de los últimos recursos de comunicación en casos extremos. Su importancia en el ámbito mundial y número de seguidores día a día aumenta, contribuyendo a la formación de ligas de radioaficionados locales. Por otro lado, el conocer cómo diseñar tipos de antenas de radio afición también es de gran interés para no solo las comunidades que conforman las ligas, sino también se incrementa en el ámbito académico, particularmente en la práctica en un sistema de comunicación y su consideración en entornos de emergencias y desastres.

Esta investigación evidencia como se planteó el diseño de un prototipo de antena a partir de elementos de facil acceso y posterior a su construc- ción, las pruebas de transmisión y recepción para su análisis espectral en un entorno local.

En primer lugar, brevemente se explica la importancia de la radio afición en las comunicaciones, luego se lleva a cabo una descripción sobre el tipo de antena escogida y los métodos empleados para su construcción, posteriormente se realiza un análisis comparativo de las diferentes pruebas llevadas a cabo bajo diferentes potencias de transmisión y distancias en un escenario local.

Palabras clave:

antena, comunicación, potencia, radio afición, recepción, transmisión, Yagi-Uda. (c)

SOMERIIHHSHESERVE
Los textos publicados en esta revista están sujetos -si no se indica lo contrario- a una licencia de Reconocimiento 4.0 Internacional de CreativeCommons. La licencia completa se puede consultar en https://creativecommons.org/licenses/by/4.0/deed.es_ES.

Aprobado evaluador interno: 1/11/2019

Aprobado evaluadores externos: 1/11/2019 


\section{Introducción}

Hoy en día las telecomunicaciones en emergencia son reconocidas como un punto clave en la prevencióny a cón comoun punto clave en la preven2003 la Unión desastres [1]. Sibiendesde elaño (UIT) ha hablo del Servicio de Telecomunicacio(E) nes en Emergencias (Emergency Telecommunication Service - ETS) [2], este, en la mayoria de los países, no se ha implementado dentro de sus redes públicas [3]

La implementación del ETS cuenta con mayores avances en cuanto a infraestructura. Ello se evidencia en el caso de Estados Unidos mediante el monitoreo y control, derivado del desastre generado por el Huracán Katrina [4]. Por lo tanto, gran número de paises de América Latina han venido implementando redes de comunicaciones en emergencias, concepto que simplemente se trata de redes de radio aficionados que apoya en situaciones de emergencias; sin embargo, esto no tiene los mismos resultados que los ETS por varias razones. Primera, porque es una red privada; segundo, porque es restringida al ser de usuarios que hagan parte de algunas instituciones como: La Cruz Roja, Bomberos, Policía etc. De este modo, es notorio en el mundo que las implementaciones de los ETS sean muy escasas, siendo así tan importantes en la actuación ante situaciones de desastre [5].

De hecho, en la última década, Latinoamérica ha atravesado escenarios de emergencias y desastres a causa de fenómenos naturales o fallas humanas; no obstante, poblaciones han aunado esfuerzos para diseñar protocolos de respuesta ante estos inconvenientes [6]. A pesar de tal medida, es necesario revisar fortalezas y debilidades en estas respuestas, considerando que sean inmediatas y eficaces, sin duplicar cargos y funciones, organizadas y sin oca [7]. En Colombia han ocurrido, durante los últimos años, catástrofes naturales en los cuales los radio aficionados intervienen para lograr comunicación en el menor tiempo posible a fin de establecer una búsqueda de apoyo. Lo anterior debido a que las comunicaciones cableadas y no guiadas probablemente colapsan a raíz de: no contar con la energía suficiente, la interconexión en uno de sus nodos, y porque el país no se encuentra preparado para enfrentar un momento de adversidad (en términos de logística) $\mathrm{ni}$ tampoco para poner en funcionamiento medios de comunicación alternos o de reserva. [8]

Ejemplo de desastres ocurridos en el país, donde las comunicaciones han estado en riesgo y ha existido una participación de radioaficionados, son: la erupción del volcán del Ruiz y la desaparición del municipio de Armero (1.985), el deslizamiento de Villatina en Medellín (1.987), el terremoto de Armenia (1.999), las inundaciones del sur del Atlántico (2010) [8] y la reciente avalancha en Mocoa - Putumayo (2017).

En función de eficacia en sistemas de radiocomunicaciones en emergencias y desastres, la preparación se logra si las organizaciones, los equipos de trabajo y las instituciones mantienen un ciclo secuencial entre actividades como las siguientes: planificación, organización, capacitación, equipamiento, ejercitación, evaluación y acciones de mantenimiento correctivo y preventivo. Además de contar con procedimientos operativos y de simulacros hacia la atención de emergencias y desastres [0]

De manera similar, la construcción de escenarios para la transmisión y recepción de datos (mediante antenas de tipo radioaficionado) podría presentarse como una estrategia pedagógica que posibilite el afianzamiento de los conocimientos a través de la práctica. Ello integra diferentes conceptosy teorias empleadas en los sistemas de comunicación.

Por lo anterior, existen iniciativas desde la academia para que estudiantes tomen conciencia de la importancia de las radiocomunicaciones en emergencias y desastres. De ahí que sea pertinente conocer la construcción de antenas con elementos a la mano y aplicar la teoría de su funcionamiento, todo con mi- ras a analizar su frecuencia de trabajo, su diagrama espectral y las pérdidas de propagación en un escenario local.

En ese sentido, esta investigación es experimental y de corte cuantitativo cuya variable dependiente son las pérdidas de potencia y la independiente la distancia.

Por ende, se diseñó una antena Yagi de mano para ure frecuencia de $850 \mathrm{MHz}$ en banda UHF. Dentr Vanda UHF. Dentro del analisis de perdidas, el problema de las tierras se soluciono elevando las antena a más de 40 centimetros de sulo. En trabajos futuros se podría implementar el analisis de patron de radiación para las antenas diseñadas, tambien el analisis espectro para espacios abiertos y a mayores distancias.

Así pues, el presente documento (posterior a esta introducción) se divide en la sección de la perspectiva o referente teórico donde se describen los conceptos que fundamentan la investigación. Luego, la perspectiva metodológica donde se incluyen la estructura en fases para su desarrollo y, posterior, se presentarán los resultados encontrados respecto al análisis de pérdidas según diferentes frecuencias y distancias. Finalmente, están las conclusiones del estudio junto con las recomendaciones futuras $y$, al cierre, las referencias en cuales se encontrará el soporte bibliográfico.

\section{Referente teórico}

A través de la historia la humanidad ha tenido la necesidad de mantener comunicación entre diferentes puntos, dentro y fuera de la tierra. donde se encuentre ubicada una comunidad o en su defecto individuo. Aun a merced de las circunstancias que pudiesen llegar a presentarse, tales como: emergencias, desastres y catástrofes.

Para los escenarios de comunicaciones por radio, sean locales 0 remotos, es importante tener en cuenta algunos términos básicos que a continuación son explicados.

\section{Proceso de comunicación}

Es el procedimiento mediante el cual se transmite una información entre emisor y receptor de forma local o a distancia. Aquí los elementos que lo componen son: emisor, quien es el que envia o transmite la información (puede ser un individuo, grupo poblacional o máquina); receptor, que es quien recibe o a quien se le entrega la información (puede ser un individuo, grupo poblacional o máquina). el código compuesto por signos o simbolos, el cual sirve para codificar el mersajes y l medio o canal desde para compde puede ser guiado o no guiado y constituye el camino por elque se va transmitiendo el mensaje (Ver Figura 1).

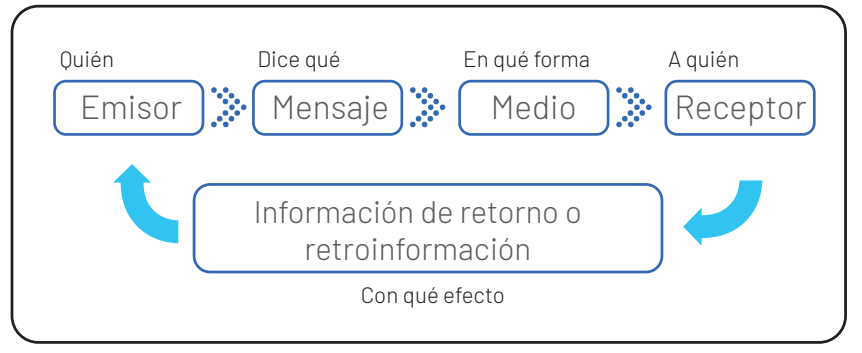

Figura 1. Proceso de la Comunicación [9]

\section{Radioafición}

La radioafición, más que un interés, es un servicio cuyo propósito es "la auto - instrucción, la intercomunicación y las investigaciones efectuados por radioaficionados" [7].

Quienes la conforman puede ser cualquier persona siempre y cuando tenga la voluntad y el deseo de adquirir algunos conocimientos básicos (Ver Figura 2). Así mismo, los radioaficionados enfocan parte de sus esfuerzos día a día, no sólo por el gusto o por el intercambio que se puede dar al hacer parte de este grupo de aficionados, sino porque reconocen la importancia que esto puede tener bajo cualquier eventualidad posible. 


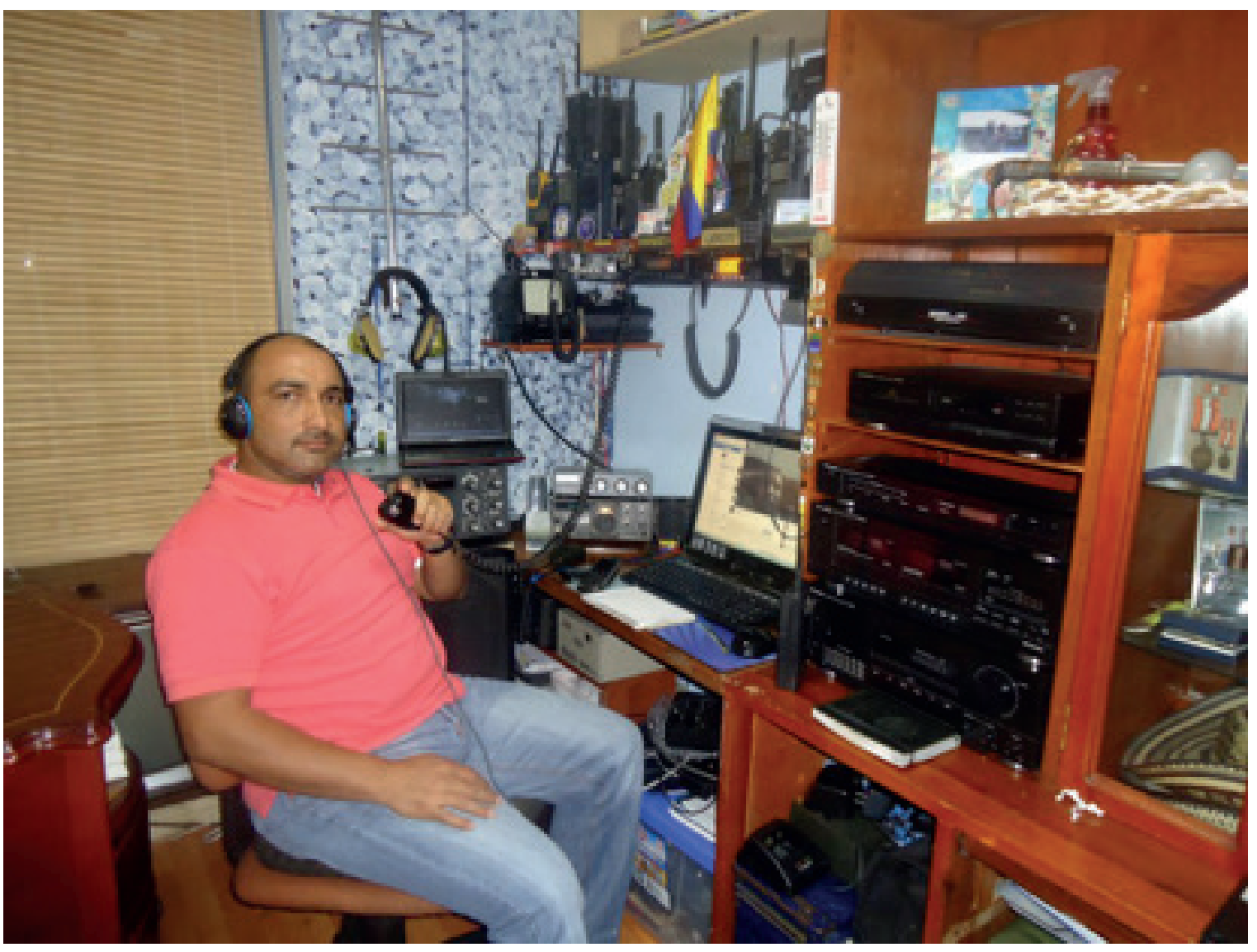

Figura 2. Radioaficionado en la ciudad de Bogotá.

La radioafición también es "un medio para que las personas se desarrollen en el ámbito cientifico y tecnológico" [10], bajo el uso de bandas del espectro radioeléctrico, las cuales tiene las siguientes características para la comunicación por radio:

- Banda exclusiva: solo los radioaficionados pueden usarlas.

- Banda de uso compartido: los radioaficionados pueden compartirlas con otros usua rios.

- Banda compartida primaria: los radioaficionados son prioritarios en su uso.

- Banda compartida secundaria: los radioaficionados deben abstenerse de interferir a los usuarios con banda compartida primaria [10].
Antena Yagi-Uda

Este tipo de antena es un arreglo de dipolos con máxima dirección de radiación a lo largo de su eje principal. Su ancho de banda es estrecho y su impedancia de entrada es baja. Los dipolos de dicho artefacto se organizan según cálculos en función de la longitud de onda, colocados de forma paralela entre ellos [11].

Uno de sus dipolos es activo, el cual es alimentado de forma directa; los demás son pasivos o elementos parásitos. Solo el dipolo ubicado detrás del activo sirve de reflector, y los restantes, ubicados delante del dipolo activo o radiador, hacen las veces de directores [11] como se observa en la Figura 3 .

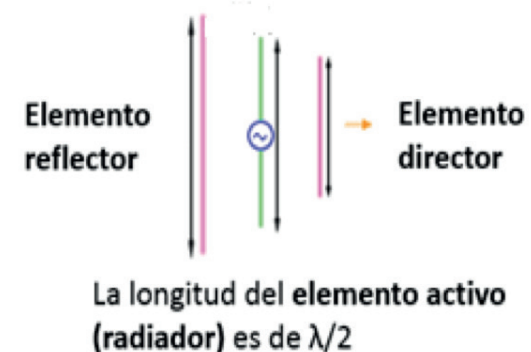

Figura 3. Elementos que componen una antena Yagi-Uda.
Este tipo de antena se utiliza para la transmisión de señales de televisión y también en comunicaciones por radio frecuencia.

\section{Decibelio}

Resultado logarítmico de la relación entre dos magnitudes, sean de origen acústico, eléctrico o entre la que se estudiay analiza una situación de referencia. Su unidad es el belio (B), que equivale a 10 decibelios (o 10 veces $B$ ), representando un aumento de potencia de 10 veces sobre la magnitud de referencia [12].

\section{Metodología}

La investigación experimental de corte cuantitativo se llevó a cabo mediante una serie de fases, las cuales se pueden apreciar a continuación:

- Fase uno: Diseño de la antena.

- Fase dos: Caracterización y ancho de banda.

- Fase tres: Adecuación, pruebas y análisis espectral.

\section{Fase uno: diseño de la antena}

En esta se consideró que la antena a diseñar cumpliera con las siguientes caracteristicas: portabilidad, bajo costo (materiales para su construcción) y maniobrabilidad.

A partir de lo anterior, se seleccionó la antena Yagi-Uda de 3 elementos con una longitud de 70 centímetros, en banda UHF (Ultra High Frequency).

Los materiales utilizados fueron: cable RG-58, barras de aluminio, tapones plásticos, tornillos, conector BNC macho y balun. Esto fue suficiente para que en su diseño se tuviesen en cuenta los tres elementos que se muestran de la Figura 4 bajo el estándar aproximado que allí se evidencia, con un lambda $(\lambda)$ o longitud de onda de 60 centímetros.
Elemento reflector

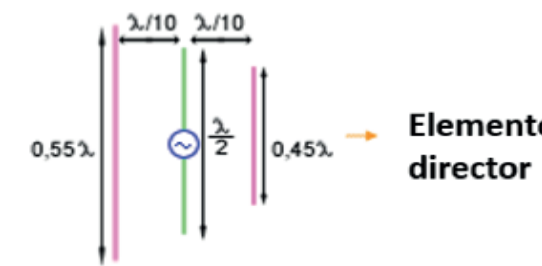

La longitud del elemento activo (radiador) es de $\lambda / 2$

Figura 4. Cálculos para el diseño de antena Yagi-Ud

Para el elemento reflector, la longitud total es de 33 centímetros, la longitud del elemento radiador de 30 centímetros y el elemento director 27 centímetros. El prototipo de antena Yagi-Uda construida se aprecia a continuación (Ver Figura 5)

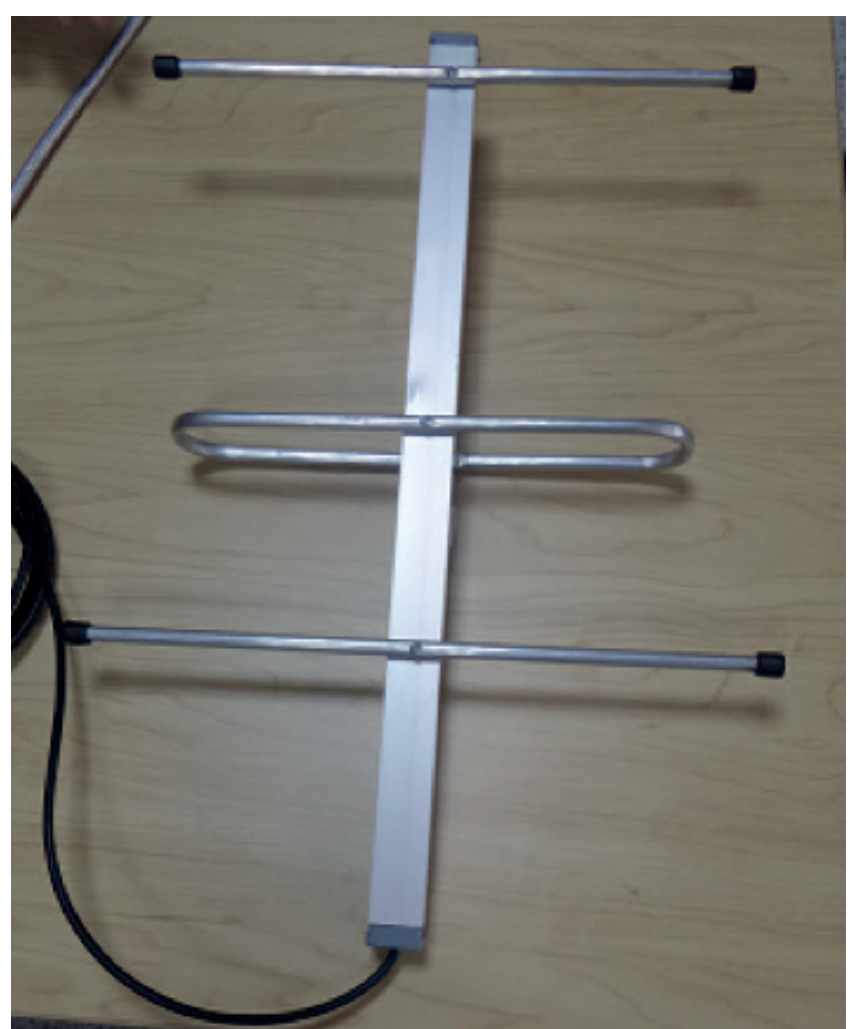

Figura 5. Prototipo antena Yagi-Uda diseñada.

Fase dos: caracterización y ancho de banda

El balun, fue necesario para su conexión hacia el analizador de espectro y asi identificar la frecuencia de la antena resultante, la cual se ubica aproximadamente en 853,2 MHz (Ver Figura 6). Esta antena se utilizará como antena receptora y servirá en las pruebas a diferentes distancias. 

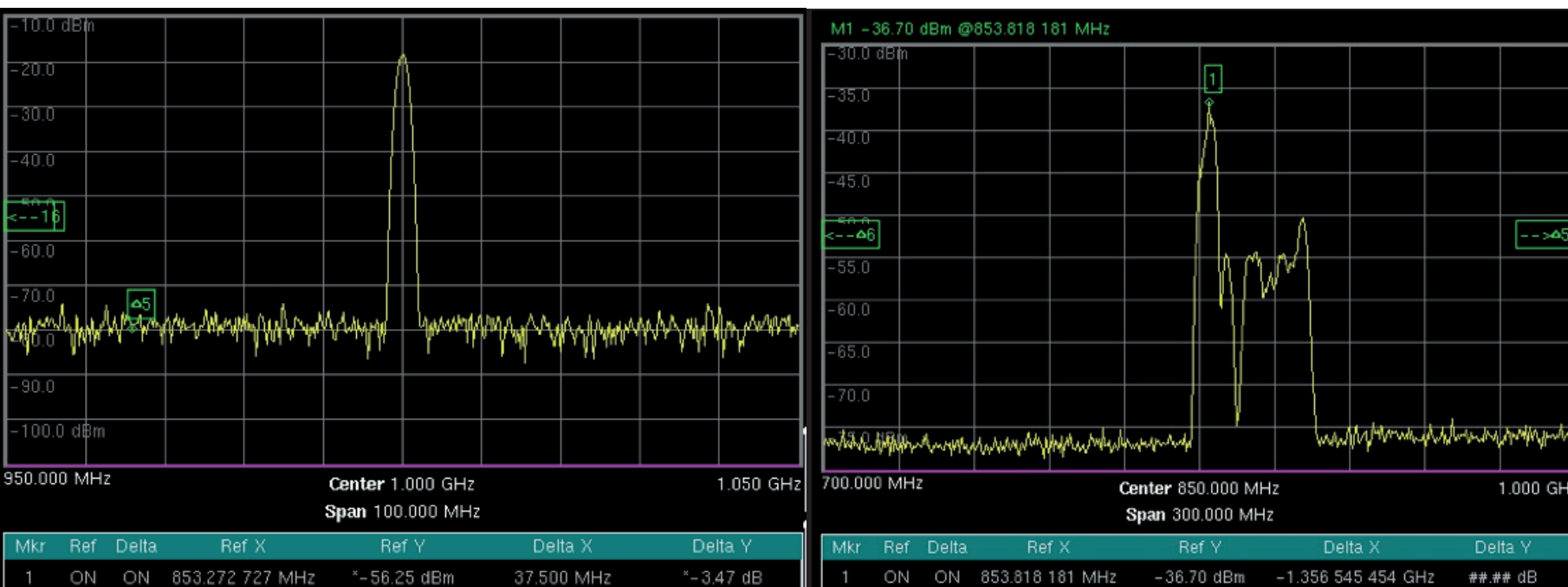

Figura 6. Frecuencia resultante antena de radioafición diseñada.

El spam determinado para las pruebas fue de $100 \mathrm{MHz}$ y se escogieron tres frecuencias de estudio: $950 \mathrm{MHz}$, $1000 \mathrm{MHz}$ y $1050 \mathrm{MHz}$.

La antena de transmisión, conectada al radio transmisor también es de tipo Yagi, de 6 elementos previamente construida (Ver Figura 8).

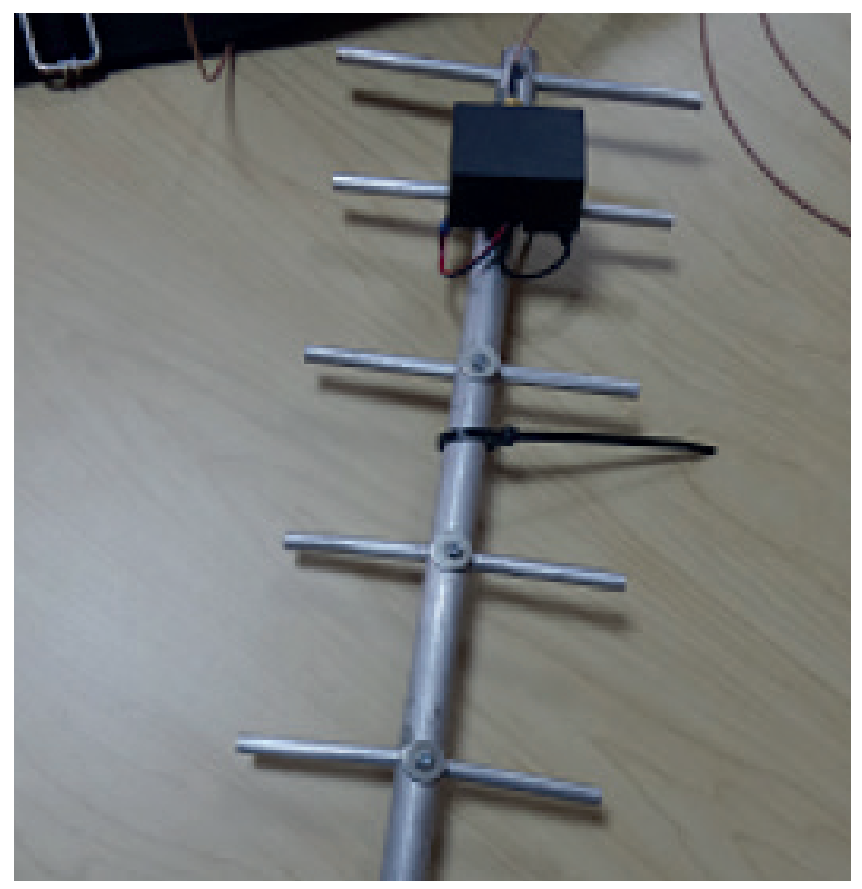

Figura 7. Antena Yagi previamente construida

Al identificar su frecuencia central, se obtuvo un resultado de $853,8 \mathrm{MHz}$ (Ver Figura 8), similar a la frecuencia de la antena de radio afición diseñada como objeto de este estudio.
Figura 8. Frecuencia antena Yagi previamente construida

Fase tres: adecuación, pruebas y análisis espectral

Dicha fase contempló las distancias seleccionadas para las pruebas de transmisión/recepción. Estas fueron desde 1 metro hasta 8 metros, con intervalos de 1 metro, con potencias de transmisión de: $10,5,0,-5,-10,-15,-20,-25,-30,-35$, $-40,-45$ y $-50 \mathrm{dBm}$; pero para el análisis de resultados se establecieron: $10,-5,-20,-35$ y -50 $\mathrm{dBm}$. Es decir, cinco potencias diferentes con intervalos de $15 \mathrm{dBm}$.

Para realizar la transmisión se situaron las antenas a una altura de 40 centímetros con respecto al suelo, obteniendo resultados óptimos en la recepción y visualización de datos mediante el analizador de espectro.

Para cada una de las distancias se desarrollaron pruebas con diferentes potencias de transmisión a la frecuencia de $950 \mathrm{MHz}, 1000 \mathrm{MHz}$ y 1050 $\mathrm{MHz}$

En efecto, este procedimiento se efectuó aumentando la distancia de a metro, hasta llegar a los 8 metros de distancia, obteniendo 13 (trece) potencias de transmisión por cada una de las 3 (tres) frecuencias centrales, por las 8 (ocho) distancias, para un total de 312 datos obtenidos. Sin embargo sólo 120 fueron objeto de estudio para los resulta debido a las 5 potencias de transmisión escogidas.

\section{Resultados}

Luego de tabular los datos obtenidos se realizaron las gráficas correspondientes a cada frecuencia ( $950 \mathrm{MHz}$, $1000 \mathrm{MHz}$ y $1050 \mathrm{MHz}$ ) por cada una de las 8 distancias para las potencias de transmisión: $10 \mathrm{dBm},-5 \mathrm{dBm},-20 \mathrm{dBm},-35 \mathrm{dBm}$ y $-50 \mathrm{dBm}$.

\section{Cálculos para pérdidas}

Para realizar estos cálculos de pérdidas de transmisión / recepción de una manera más detallada, fue necesario tener en cuenta que la medida se tomó desde la salida del transmisor, adicional la captura de datos se realizó entre los extremos, tomando como referencia 1 metro (valor mayor de potencia) y 8 metros de distancia (valor menor de potencia).

Por lo anterior, la fórmula (1) permitió calcular las pérdidas en cada punto.

Pérdidas en $d B$ en cada punto $=d B m_{\text {(iin }}+d B m_{\text {(owt) }}(1)$

Siendo:

$d B m_{(i n)}=$ Potencia del transmisor $y$

$d B m_{\text {(out) }}=$ Potencia del receptor medida en cada punto

Luego de calcular en cada punto el valor de pérdidas en $\mathrm{dB}$, fue necesario convertir el valor a Watts, para lo cual:

$P(W)=10^{d B}{ }_{10}(2)$

Posteriormente, se calculó la diferencia entre los puntos de 1 metro y de 8 metros:

Pérdida total $(W)=P\left(W_{(1 \text { metro }}\right)-P\left(W_{-8 \text { metros }}\right)$ (3)

Al obtener este resultado, se realizó la conversión de Watts a dB para conocer las pérdidas.

$P(d B)=10 \log \quad(W) \quad(4)$

Por último, se conviertió a dBm para obtener las pérdidas totales del sistema, mediante las siguientes fórmulas:

$P(d B m)=P(W){ }_{l m W}(5)$
Prueba 1: Potencia de transmisión $10 \mathrm{dBm}$

Cuando se ajusta el radio transmisor a una potencia de $10 \mathrm{dBm}$, realmente se está transmitiendo a $10 \mathrm{~mW}$, el comportamiento de la Figura 11. Entonces se observa que, para las tres frecuencias utilizadas, al aumentar la distancia la potencia disminuye, se tiene un valor más elevado en potencia recibida en $\mathrm{dBm}$ para la frecuencia de $1 \mathrm{GHz} 01000 \mathrm{MHz}$ en la distancia minima de 1 metroy máxima de 8 metros frente a las otras 1 metro y maxima de 8 metros frente a las otras más perdidas en Watts.

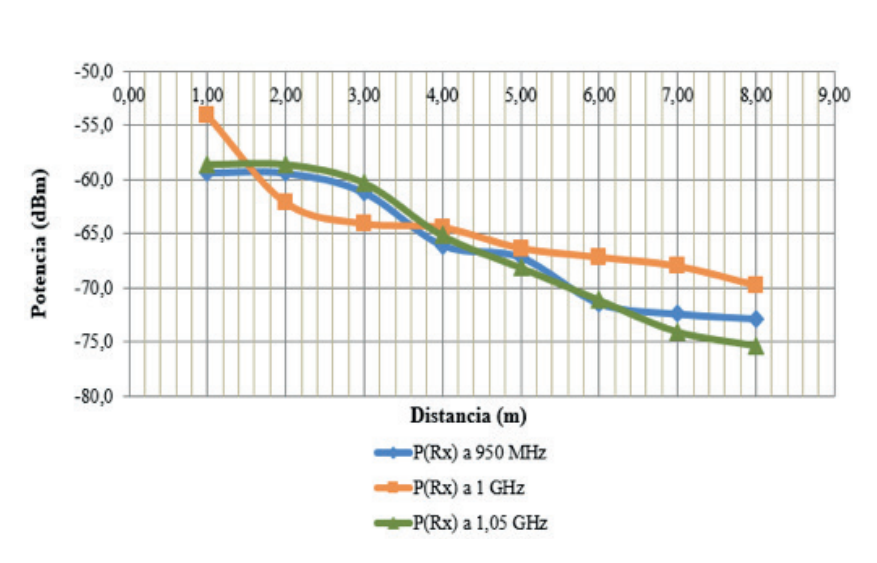

Figura 9. Distribución de Potencia de Recepción con $\mathrm{P}(\mathrm{t} x)=$ $10 \mathrm{dBm}$

Los cálculos descritos anteriormente, fueron realizados para las tres frecuencias, tal y como se aprecia en la siguiente Tabla 1.

Tabla 1.

Análisis de pérdida con $P(T X)=10 d B m$

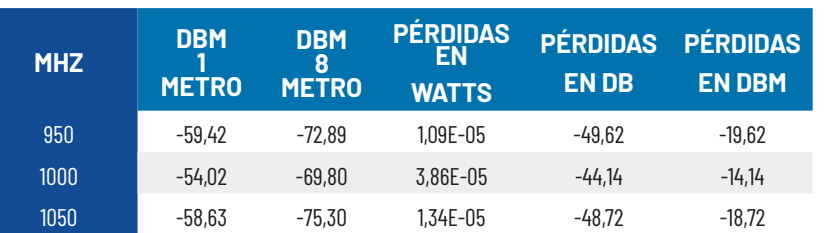

Al analizar la Tabla 1, se evidenció que para una transmisión de $10 \mathrm{~mW}$ ó $10 \mathrm{dBm}$, la pérdida en las tres frecuencias fue mínima (en el orden de los $\mathrm{mW}$ $\mathrm{o} \mu \mathrm{W}$ ), siendo cercanas entre 1 y $4 \mu \mathrm{W}$ y en $\mathrm{dBm}$ desde -14 hasta casi $-20 \mathrm{dBm}$ 


\section{Prueba 2: Potencia de transmisión} $-5 \mathrm{dBm}$

Para una potencia de transmisión de $-5 \mathrm{dBm}$, (equivalente a $0,316 \mathrm{~mW}$ ), el comportamiento de la Figura 10 evidenció qué para las tres frecuencias utilizadas, al aumentar la distancia el valor de potencia va decayendo. Nuevamente se tiene un valor más elevado en potencia recibida para la frecuencia de $1 \mathrm{GHz}$ o $1000 \mathrm{MHz}$ en la distancia mínima de 1 metro y máxima de 8 metros, pero de nuevo es la frecuencia que tiene mayor valor de pérdidas durante la distancia total.

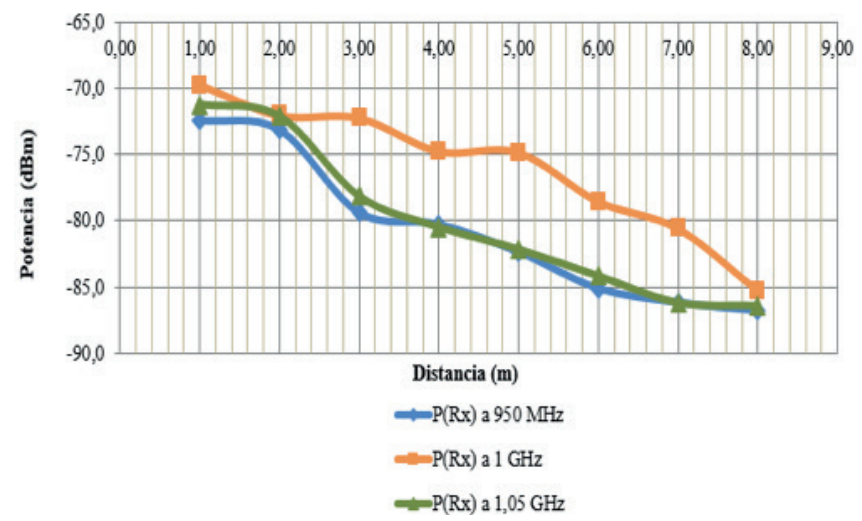

Figura 10. Distribución de Potencia de Recepción con $\mathrm{P}(\mathrm{tx})=$ Figura
$-5 \mathrm{dBm}$

Luego de analizar la Tabla 2, se obtuvo como resultado que, para una transmisión de $0,316 \mathrm{~mW}$ ó $-5 \mathrm{dBm}$, la pérdida en las tres frecuencias va a ser aún más mínima comparada con la primera prueba (en el orden de los nW), siendo cercanas entre $17 \mathrm{y}$ $32 \mathrm{nW}$ y en $\mathrm{dBm}$ desde -44 hasta casi $-48 \mathrm{dBm}$.

Tabla 2.

Análisis de pérdidas con $P(T X)=-5 d B m$

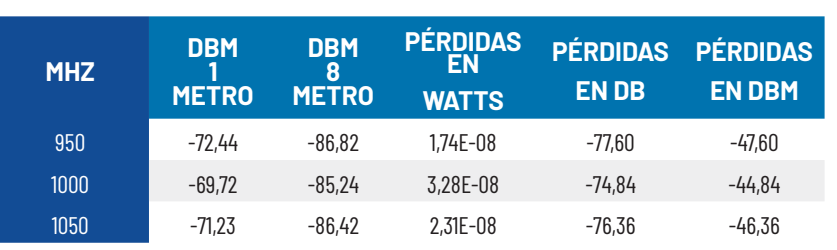

Prueba 3: Potencia de transmisión $-20 \mathrm{dBm}$

Al ajustar el radio transmisor a -20dBm, (realmente $10 \mu \mathrm{W})$, la Figura 11 registró un comportamiento si-
milar al de $-5 \mathrm{dBm}$.

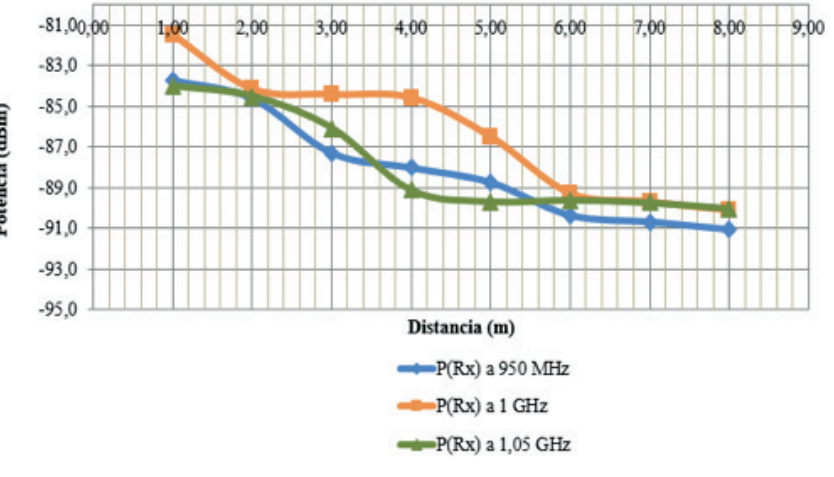

Figura 11. Distribución de Potencia de Recepción con $\mathrm{P}(\mathrm{t} x)=$ $-20 \mathrm{dBm}$

En el caso de la Tabla 3, se tiene como resultado que, para una transmision de $10 \mu \mathrm{W}$ ó $-20 \mathrm{dBm}$, las pérdidas en las tres frecuencias van a ser aún más mínimas comparada con la primera y segunda prueba (en pico Watts o $\mathrm{pW}$ ), obteniendo pérdidas totales entre casi $30 \mathrm{pW}$ hasta $61,2 \mathrm{pW}$ y en dBm entre $-72,1$ hasta $-75,2 \mathrm{dBm}$.

Tabla 3.

Análisis de pérdidas con $P(T X)=20 \mathrm{dBm}$

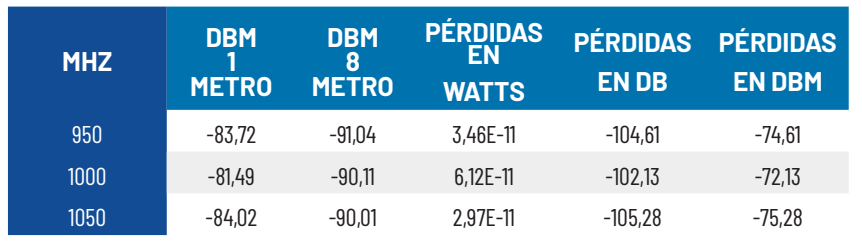

Prueba 4: Potencia de transmisión $-35 \mathrm{dBm}$

En -35dBm, (equivalente a $316 \mathrm{nW}$ ), la Figura 12 se presenta la composicion o distribución de potencia recibida durante los 8 metros en las tres frecuencias, y nuevamente al aumentar la distancia el valor de potencia disminuye.

Los valores de potencia recibidos más altos se tienen en 1 metro para $950 \mathrm{MHz}$ y en 8 metros para 1050 $\mathrm{MHz}$ perolas pérdidas totales más elevadas se presentan en $950 \mathrm{MHz}$.

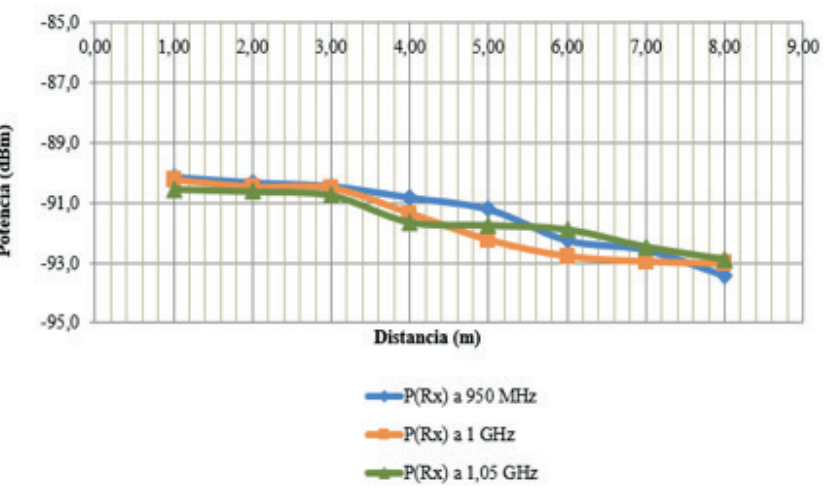

Figura12. Distribución de Potencia de Recepción con $\mathrm{P}(\mathrm{t} x)=$ Para este estudio, en la Tabla 4 se demostró que para una transmisión de $316 \mathrm{nW}$ ó $-35 \mathrm{dBm}$, las pérdidas en las tres frecuencias van a decaer aún más a comparación de los tres estudios anteriores (muy minimas cerce al orden de los fW) obteniendo pérdidas totales entre 0,117pW hasta 0,163pW yerdBm entre $-07,8$ hasta $99,3 \mathrm{dBm}$

Tabla 4.

Análisis de pérdidas con $P(T X)=-35 d B m$

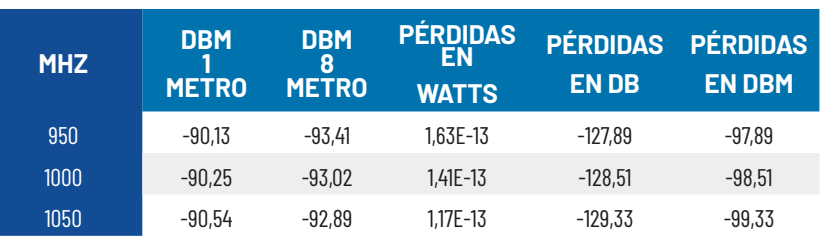

Prueba 5: Potencia de transmisión $-50 \mathrm{dBm}$

Por último, al ajustar el radio transmisor a $-50 \mathrm{dBm}$ (equivalente a 10nW), en la Figura 13 se muestra que, en la frecuencia de $1050 \mathrm{MHz}$, los valores de potencia en $\mathrm{dBm}$ recibidos fueron los más altos en el punto de inicio y fin, aunque fue la frecuencia que tuvo menos pérdidas en Watts, contrario al caso de $1000 \mathrm{MHz}$ que obtuvo las pérdidas más altas.

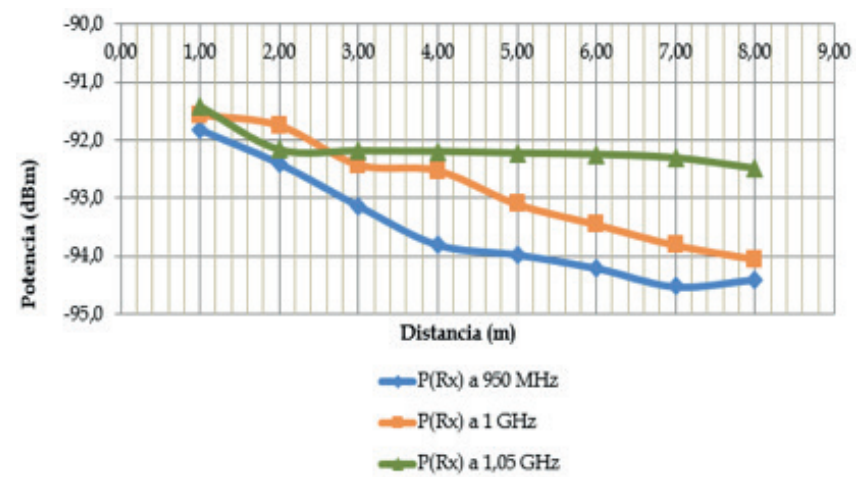

Figura 13. Distribución de Potencia de Recepción con $\mathrm{P}(\mathrm{t} x)=-50 \mathrm{~dB}$
La Tabla 5 muestra los resultados del último estudio, y para una transmision final de $10 n W$ o $-50 \mathrm{dBm}$, las perdidas en las tres frecuencias decrecieron aún más frente a los estudios anteriores (en orden de los fW), con resultados entre los 1,5 fW hasta los $3 \mathrm{fWy}$ en $\mathrm{dBm}$ entre los $-115,1$ hasta los -118 .

Tabla 5.

Análisis de pérdidas con $P(T X)=-50 \mathrm{dBm}$

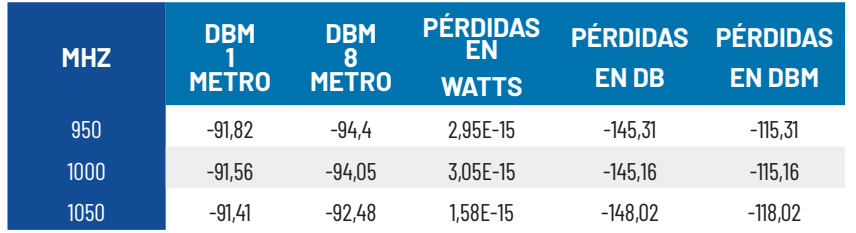

\section{Conclusiones}

Por un lado, bajo el estudio de transmisión / recepción mediante el uso de antenas de radio afición se comprueba que, a mayor distancia, la curva de potencia recibida decrece. Esto se cumple para los 5 valores de potencia transmitida y bajo las 3 frecuencias de uso objeto de este trabajo.

Por otro, se evidencia también que, al ir disminuyendo el valor de potencia de transmisión, a su vez el valor total de perdidas disminuye desde el orden de $\mu \mathrm{W}$ hasta los fW.

Adicional, se comprobó que la antena de mano Yagi-Uda diseñada tenía una frecuencia específica de $853 \mathrm{MHz}$ y que funcionó correctamente durante las pruebas, permitiendo obtener diferentes valores 0 datos en el transcurso del análisis espectral durante los 8 puntos de medida. Así mismo, la aplicación de las conversiones logaritmicas de potencia fue de granvtilidad, permipotencia.
tiendo usar unidades en decibelios, dB, o Watts de

Por último, se concluye que la construcción de antenas de Radio afición es de gran utilidad para la transmisión de datos locales; sin embargo, su impacto puede trascender no solo en el campo académico sino también se podrían propiciar espacios de ciencia, tecnologia e innovación en la comunidad, a través del conocimiento y elaboración de dispositivos y antenas de radiocomunicaciones improvisadas; entre otros. De esta manera se dan avances que contribuirían como medio (más no como fin) en la implementación de las estaciones y nodos en emergencias y desastres. 
También es importante concientizar a la comunidad no sólo en el hecho de la elaboración de la antena casera, sino en su uso y la mediación de las TIC para comprender términos como la propagación de señales, uso de frecuencias, manipulación de equipos y las pérdidas que se pueden tener por espacio libre, distancia y obstáculos en el recorrido.

\section{Recomendaciones}

A corto plazo se considera continuar trabajando en otros modelos de antenas de radio afición. ReA corto plazo se considera continuar trabajandocientemente se conal una antena tipo EH de 20 metros de alcance, con una frecuencla de $15 \mathrm{MHz}$ (Ver Figura 14). Con ello se espera construir una segunda antena de este tipo para realizar la trasmisiony recepción de portadora y luego posterior analisis espectral bajo las mismas potencias y distancias empleadas en este objeto de estudio.

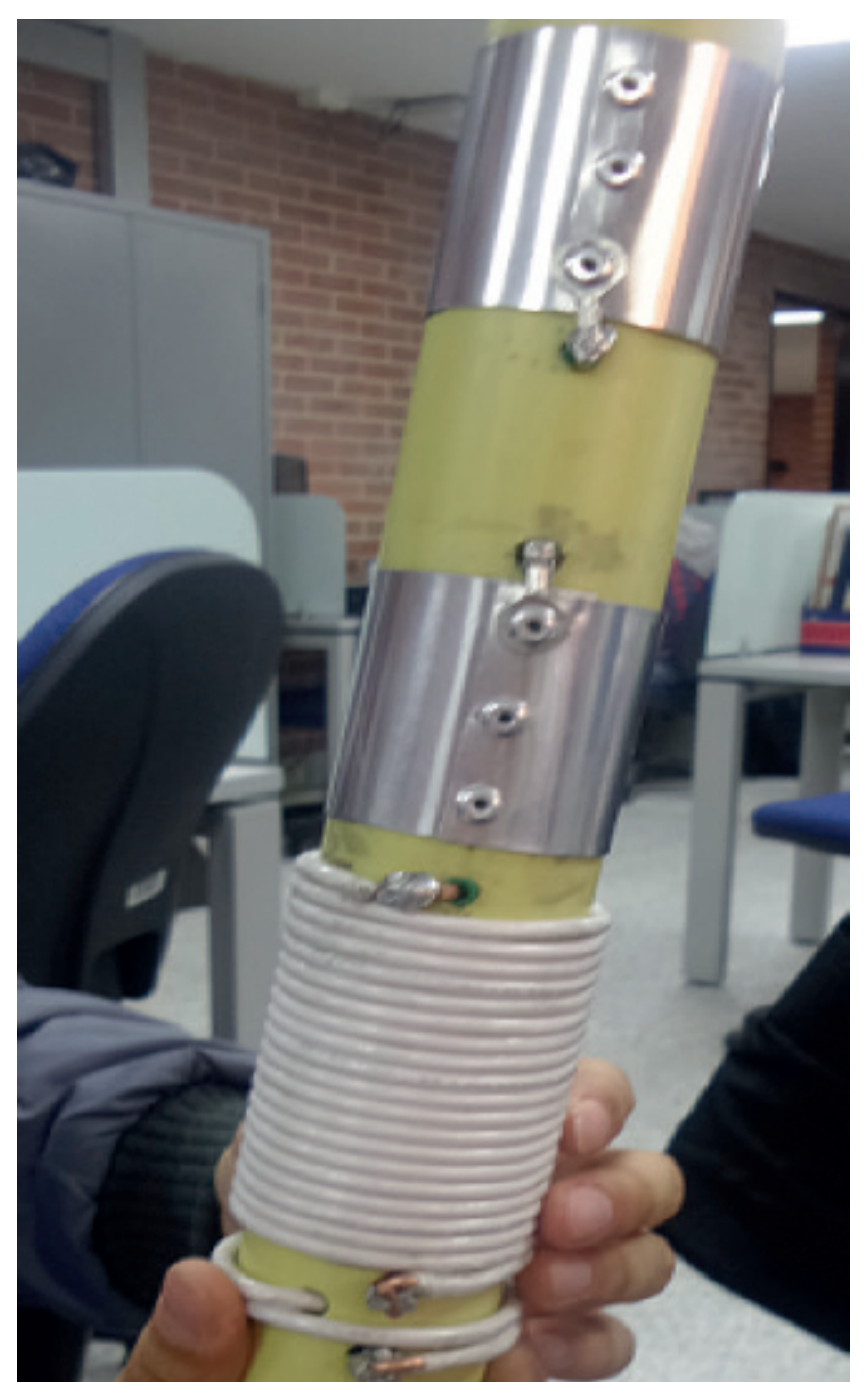

A su vez, se puede considerar incluir estudios de patron de radiación de estas antenas para conocer su comportamiento en radiación en planos de campo eléctrico y magnético, su diagrama polar y cartesiano $y$, de este modo, conocer especificamente en el espacio la directividad y ganancia de cada antena.

Para estudios a mediano plazo se pretende realizar pruebas en campo abierto, con mayores distancias de transmisión y antenas más grandes como por ejemplo son: Onmidireccional para VHF, Ouad Cúbica, Tela de Araña, Panal de Abeja, Slim Jim. Band Ha de Araña, Panal de Abeja, Slim Jim, Loop, cluir no solo la transmisión del dato de podria insino también señla de portadora sino tambien, señales digitales electrofisiológicas como por ejemplo las ya acondicionadas bajo proyectos previos como: Electrocardiográfica, Pletis-
mográfica y Oximetría de Pulso.

\section{Agradecimientos}

A la Universidad Militar Nueva Granada y Vicerrectoría de Investigaciones por financiar el Proyecto de Iniciación Científica PIC - ING - 2261. También, al programa de la Tecnología en Electrónica y Comunicaciones y su Grupo de Investigación e innovación Tecnológica en Electrónica y Comunicaciones (GI-iTEC), junto al semillero Faraday.

\section{Referencias}

[1] 0. Unidad Nacional para la Gesti\&oacute;n del Riesgo de Desastres, "Socialización Del Decreto Del Sistema Nacional De Telecomunicaciones En Emergencias", Repositorio. gestiondelriesgo.gov.co, 2015. [Online]. Available: https://repositorio.gestiondelriesgo.gov. co/handle/20.500.11762/11466. [Accessed: 17- Aug- 2019]

[2] H. Touré, "El esfuerzo de la UIT para responder a las emergencias mundiales", Itu.int, 2011. [Online]. Available: http://www.itu.int/net/ itunews/issues/2011/02/pdf/201102_01-es.pdf. [Accessed: 02- Sep- 2019].

[3] H. Folts, "Standards initiatives for Emergency Telecommunications Service (ETS) - IEEE Journals \& Magazine". 40(7), 102-107., leeexplore.ieee.org, 2002. [Online]. Available: https://ieeexplore.ieee.org/abstract/document/1018014. [Accessed: 03- Mar- 2019].

[4] T. Henderson, K. Roberto and Y. Kamo, "Older Adults' Responses to Hurricane Katrina: Daily Hassles and Coping Strategies - Tammy L. Henderson, Karen A. Roberto, Yoshinori Kamo, 2010", SAGE Journals, 2009. [Online] Avilable: https://journals, 2009ub.come]. sagepub.com/doi/ 06- Aug- 2019]

[5] 0. Unidad Nacional para la Gestión del Riesgo de Desastres, "Por primera vez, Colombia tendrá un Sistema Nacional de Telecomunicaciones en Emergencias", Repositorio. gestiondelriesgo.gov.co, 2015. [Online]. Available: https://repositorio.gestiondelriesgo. gov.co/handle/20.500.11762/11216. [Accessed: 11- Mar- 2019].

[6] A. Garijo Sánchez, "Análisis de capacidades nacionales en preparacion a emergencias en UNICEF para la region de Latinoamerica y $\mathrm{Ca}$ ribe", Riunet.upv.es, 2014. [Online]. Available: https://riunet.upv.es/handle/10251/43775. [ACcessed: 29- Apr-2019].

[7] D. Sierra and Z. Ramos, "Estado del arte de los Servicios de Telecomunicaciones de Emergencia (ETS) en el Colombia", Universidad Naciona de Colombia. Maestría en Ingeniería en Telecomunicaciones, Bogotá, pp. 1-6, 2009.

[8] A. Erazo Coronado and J. Arroyave Cabrera, "Comunicación de riesgo y de crisis en desastres de origen natural en Colombia", Congreso. pucp.edu.pe, 2014. [Online ]. Available: http:// congreso.pucp.edu.pe/alaic2014/wp-content/ uploads/2013/09/Ana-Mar\%C3\%ADa-Erazo-y-Jes\%C3\%BAs-Arroyave.pdf. [Accessed: 12- May- 2019].
12-y-Jes\%

[9] D. López Miguel, "Recursos sobre emergencias y ayuda humanitaria en Internet", Core. ac.uk, 2007. [Online]. Available: https://core. ac.uk/download/pdf/47783050.pdf. [Accessed 06- Oct- 2019].

[10] V. Pinilla Morán, R. Gress Gómez, J. Razo Pérez and G. Colloli Hernández, "Conceptos bá- sicos de la radioafición", Areunam.unam.mx, 2011. [Online]. Available:http://www.areunam. unam.mx/papime/practica01.pdf. [Accessed: 22-Mar- 2019].

[11] Gómez, L. Barrero Páez and R. Celeita, "Diseño de antenas Yagi Uda usando algoritmos genéticos", Revistas udistrital.edu.co, 2003. [Online] Avalable: https://revistasudistrital.edurco/inAvailable: https://revistas.udistrital.edu.co/index.php/reving/article/download/26 line $=1$. [Accessed: 12- Jul- 2019].

[12] G. Trujillo Rojas, A. Ortíz Ávila and N. Vásquez Díaz, "Diseño y construcción de un prototipo para un sistema de control remoto de antena de microondas", 45.5.172.45, 2019. [Online]. Available: http://45.5.172.45/handle/10819/2970. [Accessed: 04- Feb- 2019]. 